Research Article

\title{
The Simulation of a Surface Plasmon Resonance Metallic Grating for Maximizing THz Sensitivity in Refractive Index Sensor Application
}

\author{
Asmar Sathukarn (D), Chia Jia yi, Sakoolkan Boonruang, Mati Horprathum, \\ Khwanchai Tantiwanichapan, Kiattiwut Prasertsuk, Chayut Thanapirom, \\ Woraprach Kusolthossakul, and Kittipong Kasamsook
}

National Electronics and Computer Technology Center (NECTEC), NSTDA, 112 Thailand Science Park, Phahonyothin Road, Khlong Luang, Pathumthani 12120, Thailand

Correspondence should be addressed to Asmar Sathukarn; asmar.sathukarn@nectec.or.th

Received 30 September 2019; Revised 27 November 2019; Accepted 28 November 2019; Published 16 January 2020

Academic Editor: Paramasivam Senthilkumaran

Copyright ( $\odot 2020$ Asmar Sathukarn et al. This is an open access article distributed under the Creative Commons Attribution License, which permits unrestricted use, distribution, and reproduction in any medium, provided the original work is properly cited.

\begin{abstract}
Nowadays, the simplicity of both designing and fabrication process of a terahertz ( $\mathrm{THz})$ resonator-based sensing technique leads to its ongoing development. The consumable $\mathrm{THz}$ resonator needs to be easily integrated into an existing terahertz time domain spectroscopy (THz TDS) measurement system. It should also be able to be fabricated in a mass scale with a low production cost. In this work, a metal-coated surface plasmon resonance- (SPR-) based sensor is simulated and designed as a low-cost refractive index sensor utilizing rigorous coupled wave analysis (RCWA). To demonstrate our methodology, we design a gold-coated grating with a polydimethylsiloxane (PDMS) as a substrate, in order to perform quantitative analysis of gasoline-toluene mixture composition, which has a refraction index variation of 0.1 at $\mathrm{THz}$ frequency. The grating period is tuned such that its surface plasmon resonance (SPR) frequency matches with the peak frequency of the THz TDS system. Moreover, other grating parameters, i.e., a filling factor and a grating depth, are optimized to increase the sensor sensitivity and sharpen the resonance dip. High sensitivity up to $500 \mathrm{GHz} / \mathrm{RIU}$ with a refractive index resolution up to 0.01 is numerically revealed. The $\mathrm{H}$-field of the designed grating is then evaluated to indicate a strong SPR excitation. The well-developed designed grating introduces a promising, low-cost, and easily fabricated $\mathrm{THz}$ refractive index sensor.
\end{abstract}

\section{Introduction}

$\mathrm{THz}$ radiation is a part of an electromagnetic spectrum lying between microwaves and the far-infrared radiation (FIR). This region has frequencies ranging from 0.1 to $10 \mathrm{THz}$ and wavelengths from $3 \mathrm{~mm}$ to $0.03 \mathrm{~mm}$. THz radiation possesses properties inherited from both microwaves and IR. It is able to penetrate nonpolar materials, exhibits a high sensitivity towards moisture, and provides a specific response for each different material. At the same time, $\mathrm{THz}$ radiation is marked as safe to be used in a nondestructive evaluation due to its nonionizing nature. These unique properties of $\mathrm{THz}$ radiation can be utilized in many sensing applications [1] including those in the field of biology [2, 3], medical [4-7], and security $[8,9]$ and encouraging a comprehensive research and study.

The conventional $\mathrm{THz}$ sensing technique, which is usually performed by a THz TDS system, measures transmission or reflection spectral lines of molecular resonances while the molecules are illuminated with broadband $\mathrm{THz}$ radiation. This technique reaches its limit in detecting and analyzing a very small amount or a thin sample material. It requires a sufficient quantity or thickness to distinguish power or phase differences from the probing $\mathrm{THz}$ waves. The breakthrough of the detection limit could be achieved by the usage of the sensing devices [10], for example, a transmission 
line resonator [11], a metamaterial resonator [12], and a SPR sensor [13-16]. In general, all these devices could be described as a refractive index sensor detecting the minute change in a refractive index caused by a small amount of analytes placed in the active sensing region.

To enable the extensive uses of the $\mathrm{THz}$ sensing technique, the mass-producible, low-cost, and consumable $\mathrm{THz}$ resonators need to be easily integrated into an existing $\mathrm{THz}$ TDS measurement system. Among all the aforementioned sensing devices, the one-dimensional grating-based SPR is an attractive option due to its simplicity in both designing and fabrication process. In frequency ranges of visible light and IR, the PDMS has been introduced as a substrate material of a SPR grating to further simplify the fabrication process and reduce the fabrication cost [17-21]. For $\mathrm{THz}$ region, Nazarov et al. optimized SPR excitation on a metallic grating by varying grating parameters [22]. Subsequently, Spevak et al. and Lin et al. explored the role of material, especially semiconductors, for a THz SPR excitation [23, 24]. However, there is still a lack of research working on the lowcost $\mathrm{THz}$ SPR sensor.

In this work, a PDMS-based one-dimensional periodic rectangular $\mathrm{THz}$ SPR grating is proposed as a viable candidate of a $\mathrm{THz}$ refractive index sensor. Polydimethylsiloxane (PDMS) is low in cost, chemically stable, and biocompatible and easy for fabrication, via soft lithography or pattern transfer, supporting its use in many applications [25]. The $\mathrm{THz}$ SPR grating is numerically simulated based on RCWA. A purposed workflow for a grating structure design is created and demonstrated through an example of $\mathrm{THz}$ sensing application, i.e., the quantitative analysis of a petrochemical. This could be a guidance for designing the SPR grating in other refractive index range using the $\mathrm{THz}$ sensing technique.

\section{Numerical Method}

Surface plasmon resonance (SPR) is a coupling phenomenon of a transverse magnetic (TM) mode of an electromagnetic field of an incident wave and a collective oscillation of a free electron that is located at the boundary between the dielectric and metal [26]. These phenomena have been used in many applications, particularly, for sensing purposes [2730]. In order to excite surface plasmons, the phase matching condition needs to be satisfied. In general case for SPR, the wave vector of the incident wave $\left(k_{0}\right)$ needs to be matched with the wave vector of a surface wave $\left(k_{\mathrm{sp}}\right)$ based on the conservation of the momentum which can be expressed as

$$
k_{\mathrm{sp}}=k_{0} \sqrt{\frac{\varepsilon_{m} n_{d}^{2}}{\varepsilon_{m}+n_{d}^{2}}},
$$

where $\varepsilon_{m}$ is the permittivity of a metal and $n_{d}$ is the refractive index of a dielectric.

For the diffraction of light in the metallic grating, the wave vector of a diffracted light parallel to the grating surface is equal to the propagation constant of the surface plasmon. To be more specific, the diffraction order $m^{\text {th }}$ is required to couple to the surface wave:

$$
k_{\mathrm{sp}}=k_{0} n_{d} \sin \theta+m \frac{2 \pi}{\Lambda},
$$

where $\sin \theta$ is the angle of an incidence beam and $\Lambda$ is the grating period. Therefore, substituting $k_{\mathrm{sp}}$ from (1) into (2) gives the matching condition of SPR, which can be expressed as

$$
\begin{aligned}
n_{d} \sin \theta+m \frac{\lambda}{\Lambda} & = \pm \sqrt{\frac{\varepsilon_{m} n_{d}^{2}}{\varepsilon_{m}+n_{d}^{2}}} ; \\
\lambda & =\frac{2 \pi}{k_{0}} .
\end{aligned}
$$

Generally, the effective excitation of the surface plasmon requires $\varepsilon_{m} \gg n_{d}^{2}$. This leads to the determination of diffraction orders from the frequency response, which is controlled by the grating period $[31,32]$ :

$$
\begin{gathered}
\frac{\Lambda}{\lambda}>\frac{m}{n_{d}} ; \quad m>0, \\
\frac{|m|}{2 n_{d}}<\frac{\Lambda}{\lambda}<\frac{|m|}{n_{d}} ; \quad m<0 .
\end{gathered}
$$

In order to optimize the excitation condition, the analytical study of SPR excitation model has been developed by using a RCWA. RCWA formulation is numerically stable for solving the Maxwell equations at a single layer binary or a planar grating, especially the numerical instability in the matching of electromagnetic field at the dielectric and metal interface [33]. The Drude model is also used to derive the dispersion relation of the SPR propagating on a one-dimensional rectangular metal coated grating. Figure 1 shows the grating structure with a grating period $\Lambda$, a grating width $W$, a grating depth $H$, and a metal thickness $t_{m}$.

\section{Grating Design and Characterization}

In order to conceptualize the application of a SPR grating in $\mathrm{THz}$ sensing, the designing of an optimized grating for quantitative analysis of petrochemical and their mixture is showcased. This designed grating could be applied to other $\mathrm{THz}$ sensing application with the extended refractive index range. Because of the nonpolar nature of the petrochemical and organic solvent, $\mathrm{THz}$ spectroscopy has a great potential to be applied in analysis of liquid petrochemicals and their mixtures with an organic solvent. Researchers have studied molecular properties of petrochemical products in the $\mathrm{THz}$ range and demonstrated the qualitative and quantitative analyses of liquid petrochemicals by using a THz TDS system [34].

In this work, we numerically design a metal-coated PDMS SPR grating for improving a detection sensitivity of the $\mathrm{THz}$ TDS in analyzing a gasoline-toluene mixture, which has a refractive index lying between 1.4 and 1.5 in the frequency range of 0.2 to $2.0 \mathrm{THz}[35,36]$. The proposed experiment setup is illustrated in Figure 2. An analyte is coated on the grating surface, and the resonance 


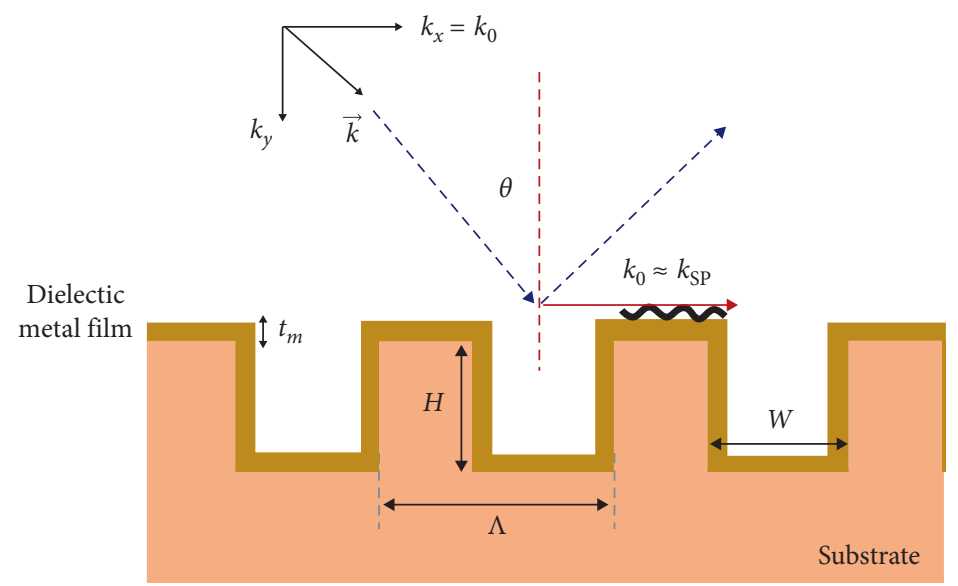

FIgURE 1: A schematic diagram of the one-dimensional rectangular metal coated grating where $\Lambda$ is the grating period, $W$ is the grating width, $H$ is the grating depth, and $t_{m}$ is the metal thickness. SPR occurs on the metal-dielectric interface when the wave vector of the incident wave $\left(k_{0}\right)$ is coupled to the wave vector of surface wave $\left(k_{\mathrm{sp}}\right)$ by the grating.

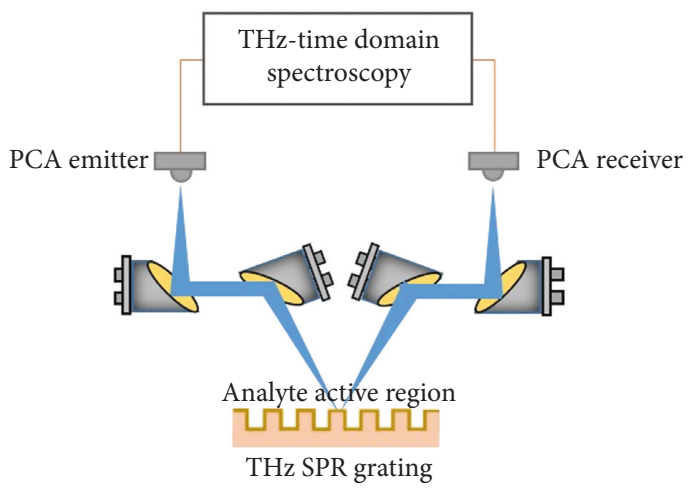

Figure 2: A THz refractive index sensing experiment setup integrating a SPR grating with THz TDS in a reflection measurement mode. The measurement should be carried out in a low-humidity environment and at room temperature to avoid strong $\mathrm{THz}$ attenuation due to moisture.

frequency shift of the $\mathrm{THz}$ spectrum is detectable resulting from the difference in the refraction index. The resonance frequency of the designed grating is aimed to be at $1 \mathrm{THz}$ or lower because most of the photoconductive antenna(PCA-) based THz TDS is limited by a bandwidth of approximately $5 \mathrm{THz}$, and the center frequency is less than $1 \mathrm{THz}[37]$.

3.1. Design and Optimization Process. The grating structure considered here, as shown in Figure 1, is composed of a PDMS substrate coated by a gold $(\mathrm{Au})$ film, which is a perfect electric conductor in $\mathrm{THz}$ range. The complex refractive indices of PDMS and $\mathrm{Au}$ film are assigned as $n_{s}=1.533-j 0.034$ [38] and $n_{m}=447-j 532$ [39], respectively. The metal thickness $t_{m}$ is an important factor for improving a sensor performance depending on the coupling configuration and structure. For example, the Au thickness affects the sensitivity of a SPR fiber grating biosensor [40]. In this study, measuring in a reflection mode, $t_{m}$ is set to be $100 \mathrm{~nm}$, which is larger than the skin depth of $\mathrm{Au}$ $\left(l_{s, A u}=74 \mathrm{~nm}\right)$ in the frequency of interest [41]. We assume that, in the case of $t_{m}>l_{s, A u}$, there is no radiative loss of resonance arising from the diffraction on the grating. Theoretically, a small incident angle of TM excitation wave would maximize the electric field on the grating structure. The incident angle of the incoming wave is set to be $10^{\circ}$, which is the minimum angle required in an actual TDS reflection measurement setup.

The geometrical parameters of a grating structure include $\Lambda, H$, and FF, which is a ratio of $W$ and $\Lambda$. Based on (3), $\Lambda$ is the first parameter to be varied in the grating optimization as it is directly related to the resonance frequency of the SPR. FF and $H$ of the initial structure are set at 0.5 and $0.5 \Lambda$, respectively. The refractive index of the dielectric, a gasoline analyte coated on the SPR grating, is taken as $n_{d}=1.4$. In this simulation, $\Lambda$ is varied from $150 \mu \mathrm{m}$ to $250 \mu \mathrm{m}$, with a step of $10 \mu \mathrm{m}$ to figure out the appropriate $\Lambda$ of the grating with a resonance frequency of 1 THz.

The result shows that the suitable $\Lambda$ is $190 \mu \mathrm{m}$. Figure 3 shows two reflectance dips existed in the range of 0.5 to $1.5 \mathrm{THz}$, located at $0.984 \mathrm{THz}$ and $1.274 \mathrm{THz}$, respectively. According to (4) and (5), these peaks corresponded to the diffraction order $m$ of 1 and -1 , respectively. This can be indicated that the resonance or plasmonic peak can be 


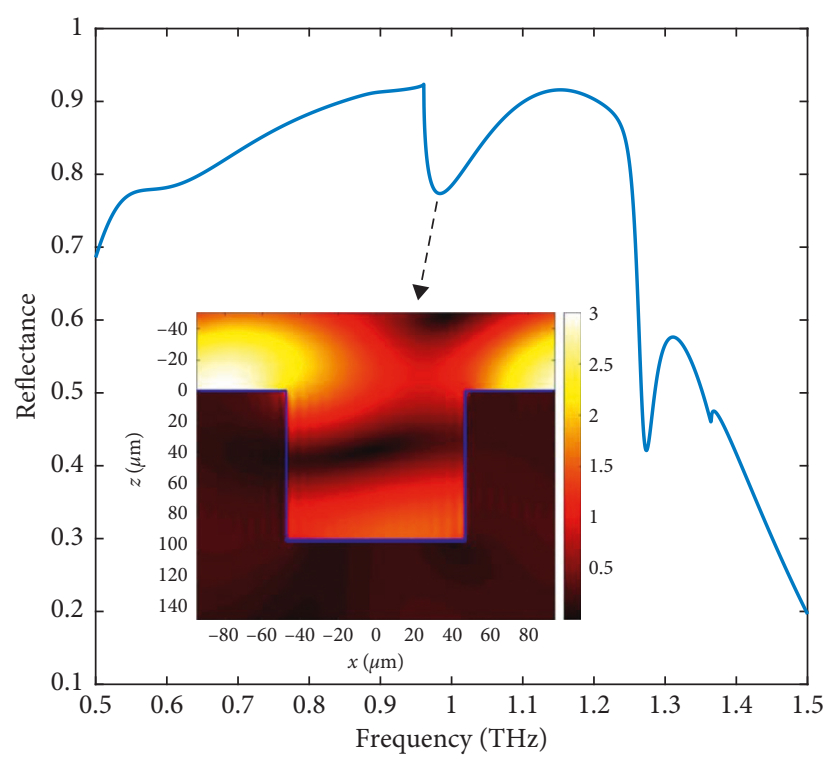

Figure 3: Reflectance of an Au/PDMS grating with $\Lambda=190 \mu \mathrm{m}, H=95 \mu \mathrm{m}, \mathrm{FF}=0.5$, and $t_{m}=100 \mathrm{~nm}$ at the incident angle of $10^{\circ}$ as a function of frequency. An inset is the $\left|H_{y}\right|$ field distribution along one unit of the grating structure.

coupled into the surface by such a designed structure. The inset of Figure 3 demonstrates the distribution of a magnetic field magnitude $\left|H_{y}\right|$ along the unit cell of the grating at the resonance frequency of $0.984 \mathrm{THz}$. The structure of the $190 \mu \mathrm{m} \Lambda$ grating needs to be further optimized by varying $\mathrm{FF}$ and $H$, which are directly related to the coupling efficiency, to minimize the reflectance of the resonance peak (reflectance $R=0.77$ at $0.985 \mathrm{THz}$ ). While $H$ is remained at $0.5 \Lambda$, the FF of a grating, which is proportional to $W$, is the second parameter to be varied. An appropriate $W$ of the grating satisfies the coupling condition and enhances the confinement of the surface plasmon electric field on the grating surface.

In the optimization process, figure of merit (FOM), defined as the ratio between sensitivity $(S)$ and the full width half max (FWHM) of a reflectance dip, is used to evaluate the performance of a grating structure [42]. The term of sensitivity $S$ is known as the rate of change of a resonance frequency $\left(f_{0}\right)$ with respect to a refraction index $\left(n_{d}\right)$ of the analyte:

$$
\begin{gathered}
S=\frac{d f_{0}}{d n_{d}}, \\
\mathrm{FOM}=\frac{S}{\mathrm{FWHM}} .
\end{gathered}
$$

In this study, FF is varied from 0.5 to 0.9 with a step of 0.05 . The optimum FF of the $190 \mu \mathrm{m} \Lambda$ grating is found in the range of 0.7 to 0.9 , which is corresponding to $W$ of 133 to $171 \mu \mathrm{m}$, giving the reflectance of less than 0.1 , as illustrated in Figure 4(a). The resonance reflection dip is shifted to a lower frequency as FF increases. The FOM plot, as shown in Figure 4(b), shows that the grating with FF of 0.8 , which has $R=0.019$ at $f_{0}=0.851 \mathrm{THz}$, is superior among all the gratings.
The parameter, $H$, is varied from 75 to $115 \mu \mathrm{m}$ with a step of $10 \mu \mathrm{m}$, to fine-tune the performance of the grating. In correspondence to the optimized $W$, an appropriate $H$ is needed to achieve a critical coupling condition whereby a reflection from the grating is suppressed and maximum power is transferred to the surface plasmon polaritons $[43,44]$. Based on the plots in Figure 5(a), $H$ of $85 \mu \mathrm{m}$ is the optimum depth for this SPR grating providing the highest FOM. $S$ of this optimized grating structure is approximately $500 \mathrm{GHz} /$ refraction index unit (RIU) in the refractive index range of 1.4 to 1.5 (maximum $S=559 \mathrm{GHz}$ / RIU at $n=1.44$ ). Figure 5(b) shows the sensitivity of the designed grating as a function of a refractive index while its inset shows the calculated resonance frequency in analyzing the gasoline-toluene mixture. As most of the commercial $\mathrm{THz}$ TDS system could achieve a spectral resolution of less than $5 \mathrm{GHz}$, the sensitivity of this SPR grating could detect the change in the refractive index of the analyte as low as 0.01 .

The reflectance of the optimized grating geometry with $\Lambda$ of $190 \mu \mathrm{m}$, FF of 0.8 , and $H$ of $85 \mu \mathrm{m}$ shows a SPR dip in the frequency range of 0.5 to $1.5 \mathrm{THz}$, with the reflectance of $3.59 \times 10^{-4}$ located at the resonance frequency of $0.869 \mathrm{THz}$ corresponding to the diffraction order $m=1$, as depicted in Figure 6(a). It can be noted that the designed structure satisfies the phase matching condition. Numerically, the $H_{y}$ field distribution on the grating surface at the resonance frequency reveals that the resonance is intense and localized at the dielectric and Au interface, as presented Figure 6(b). This indicates that the decay length of the SPR wave at the dielectric and $\mathrm{Au}$ interface is high enough to form SPR mode. In comparison, the $\left|H_{y}\right|$ intensity of the optimized structure is 4 times higher that of the initial structure, as shown in Figure 3. Table 1 shows the optimized grating parameters found in this study. 


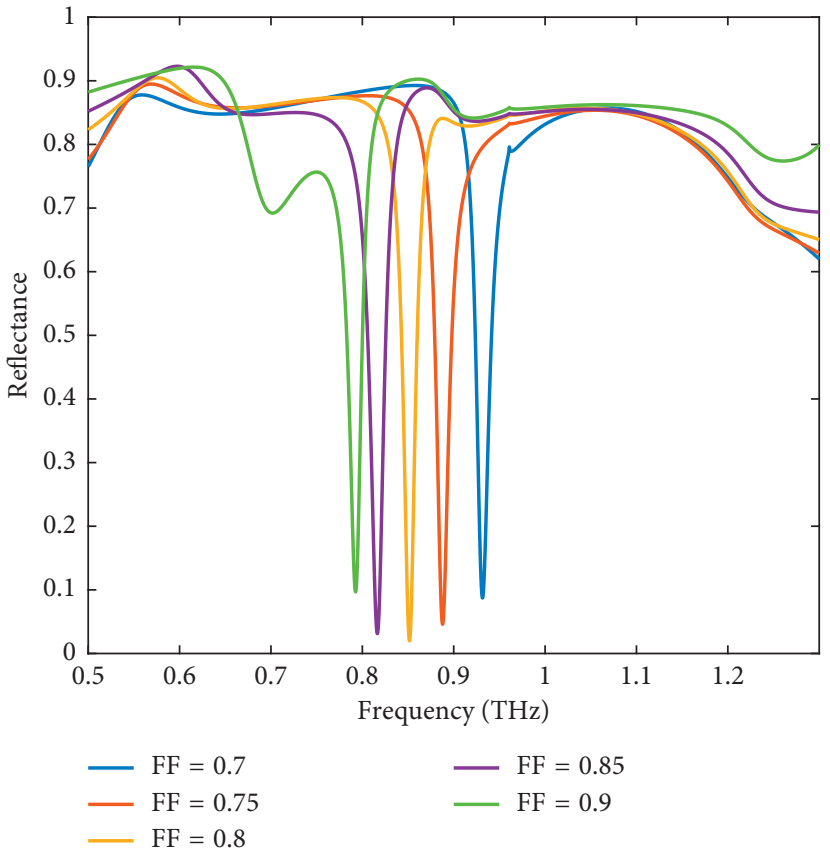

(a)

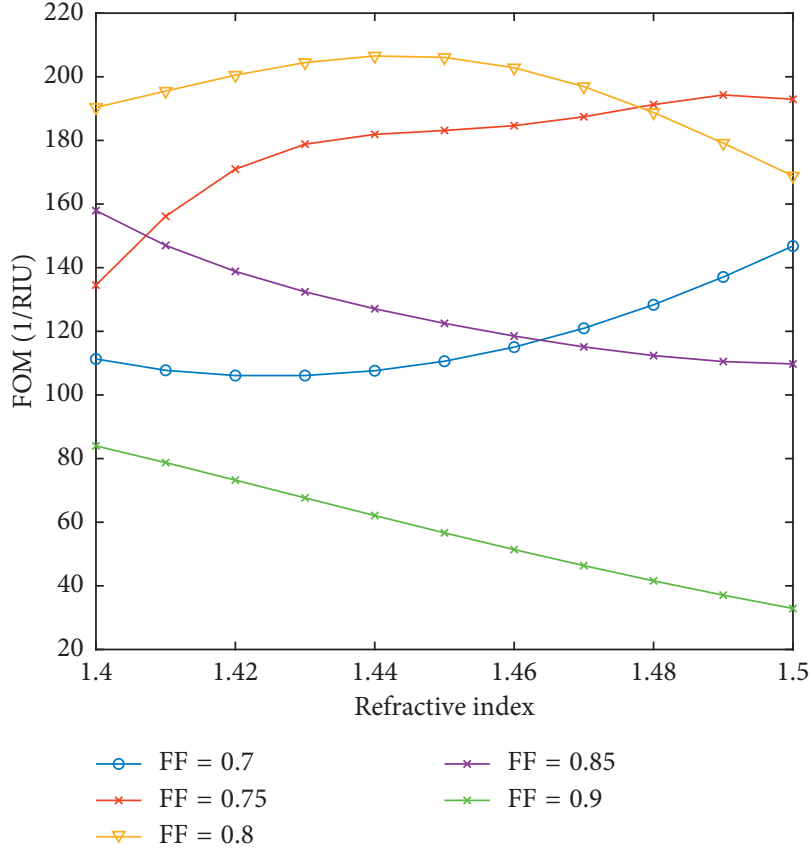

(b)

Figure 4: A variation of FF in Au-coated PDMS grating with $\Lambda=190 \mu \mathrm{m}, H=95 \mu \mathrm{m}$, and $t_{m}=100 \mathrm{~nm}$ at the incident angle of $10^{\circ}$. (a) Reflectance as a function of frequency. (b) FOM as a function of refractive index.

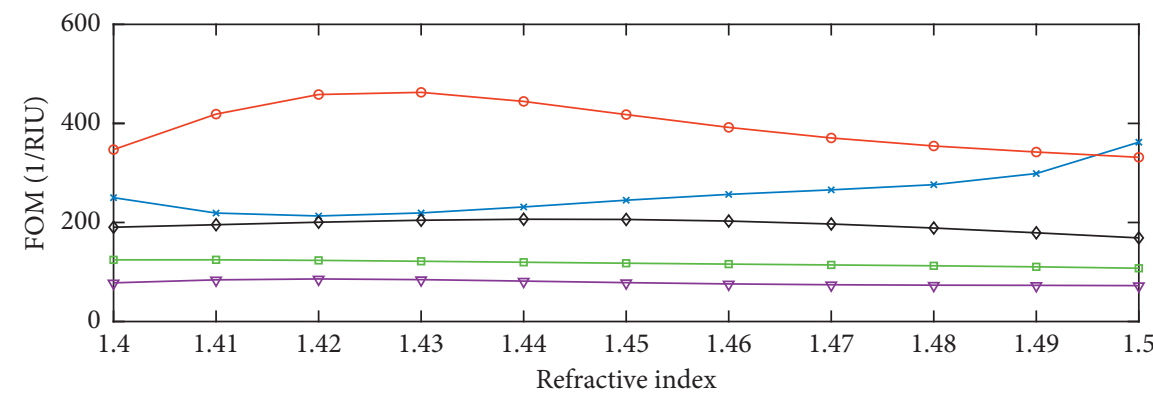

$$
\begin{array}{ll}
\multimap H=75 \mu \mathrm{m} & \rightarrow H=105 \mu \mathrm{m} \\
\multimap H=85 \mu \mathrm{m} & \rightarrow H=115 \mu \mathrm{m} \\
\multimap H=95 \mu \mathrm{m} &
\end{array}
$$

(a)

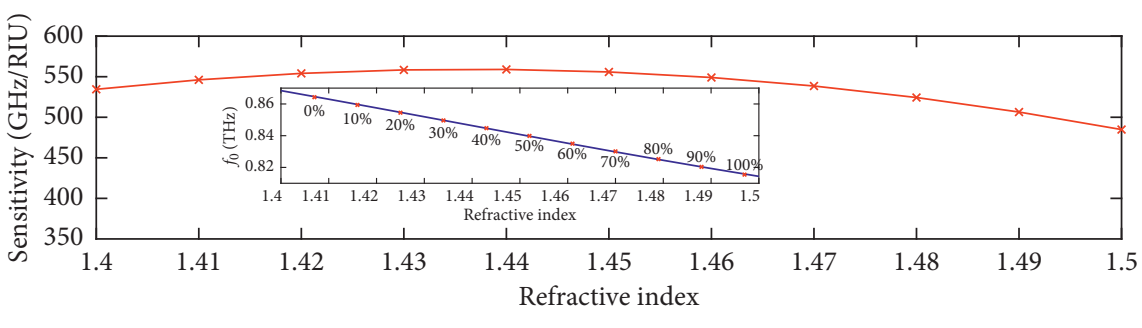

$\star \Lambda=190 \mu \mathrm{m}, H=85 \mu \mathrm{m}, \mathrm{FF}=0.8$

(b)

FIgURE 5: (a) FOM as a function of refractive index with a variation of $H$ in an Au-coated PDMS grating with $\Lambda=190 \mu \mathrm{m}$, FF $=0.8$, and $t_{m}=100 \mathrm{~nm}$ at the incident angle of $10^{\circ}$. (b) Sensitivity plot of the interested refractive range, 1.4 to 1.5 , of the optimized grating structure with $\Lambda=190 \mu \mathrm{m}, \mathrm{FF}=0.8, H=85 \mu \mathrm{m}$, and $t_{m}=100 \mathrm{~nm}$ at the incident angle of $10^{\circ}$. The inset shows the resonance frequency $f_{0}$ of this grating for different compositions of gasoline-toluene mixture. 


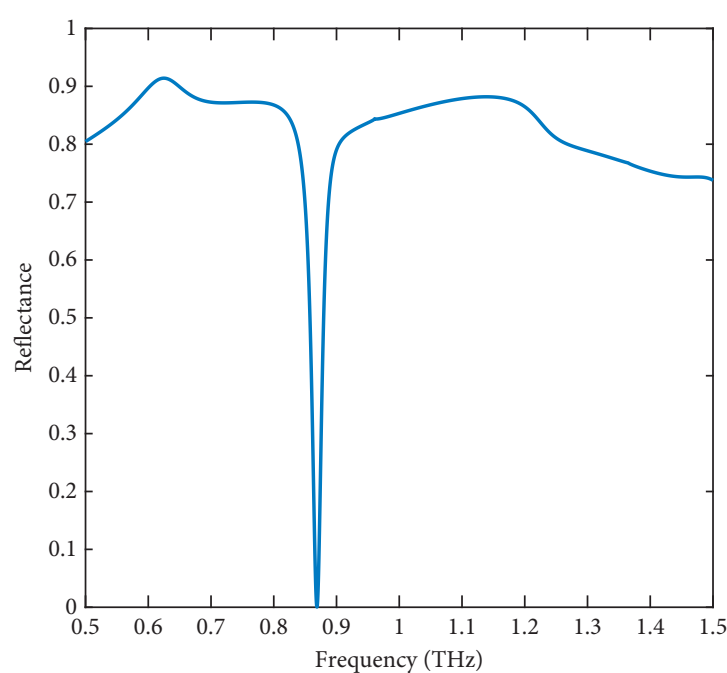

(a)

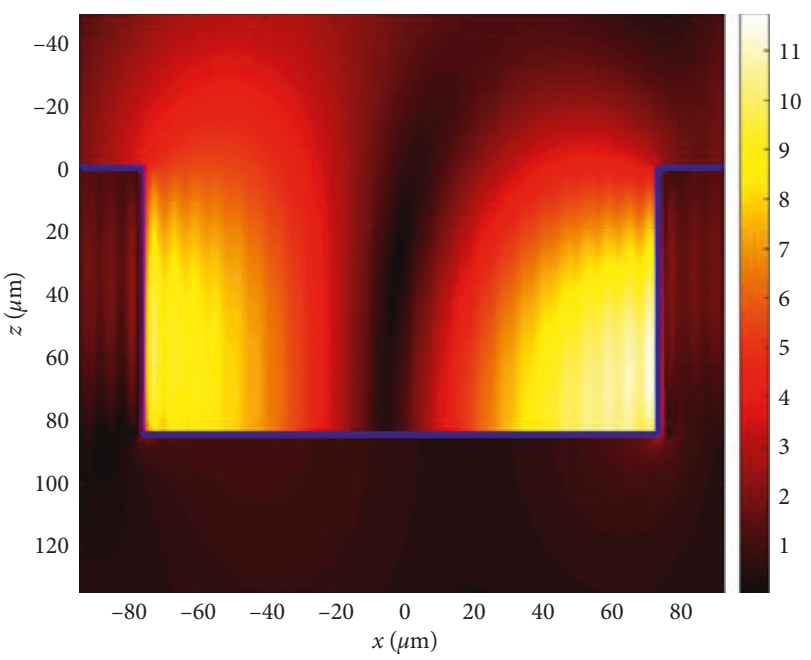

(b)

Figure 6: (a) Reflectance as a function of frequency and (b) $\left|H_{y}\right|$-field profile of a dielectric with $n=1.4$ on the Au/PDMS grating with $\Lambda=190 \mu \mathrm{m}, H=85 \mu \mathrm{m}, \mathrm{FF}=0.8$, and $t_{m}=100 \mathrm{~nm}$ at the incident angle of $10^{\circ}$.

TABle 1: The optimized grating parameters used in this study.

\begin{tabular}{lcc}
\hline Parameter & Range & Optimized value \\
\hline Grating period $(\Lambda)$ & $150-250 \mu \mathrm{m}$ & $190 \mu \mathrm{m}$ \\
Grating width $(W)$ & $133-171 \mu \mathrm{m}$ & $152 \mu \mathrm{m}$ \\
Filling factor $(\mathrm{FF})$ & $0.5-0.9$ & 0.8 \\
Grating depth $(H)$ & $75-115 \mu \mathrm{m}$ & $85 \mu \mathrm{m}$ \\
\hline
\end{tabular}

\section{Conclusion}

In this study, we have proposed a parameter design methodology for a one-dimensional periodic rectangular metallic grating design to be used in a $\mathrm{THz}$ refractive index sensor application. The sensor detects the refractive index of the coating analyte by using a $\mathrm{THz}$ TDS system to measure the shift of the grating surface plasmon resonance frequency. Our design methodology begins by varying the grating period in order to tune its resonance frequency in presence of the analyte to match the peak power frequency of the $\mathrm{THz}$ TDS system. Then, other relevant grating parameters of its $\mathrm{FF}$ and $H$ are varied to maximize the detection FOM.

The design process is demonstrated in which Au-coated PDMS-based grating is numerically simulated with a purpose of quantitatively analyzing gasoline-toluene mixture composition. The optimized SPR grating presents a sensitivity of $500 \mathrm{GHz} / \mathrm{RIU}$ resulting in a refractive index detection resolution of $0.01 \mathrm{RIU}$, which is superior to a direct measurement method. A further experimental verification of our grating design is still needed as a future work. The proposed structure could be fabricated via a standard PDMS casting and curing method following by a thin Au film layer deposition with a conventional metal deposition technique. Furthermore, the SPR grating could be utilized in other refractive index range by applying such a $\mathrm{THz}$ sensing technique.

\section{Data Availability}

The data used to support the findings of this study are available from the corresponding author upon request.

\section{Conflicts of Interest}

The authors declare that they have no conflicts of interest.

\section{Acknowledgments}

This work was financially supported by the National Electronics and Computer Technology Center (NECTEC), NSTDA, Thailand, under a funded project (P1750977).

\section{References}

[1] M. J. Fitch and R. Osiander, "Terahertz waves for communications and sensing," Johns Hopkins APL Technical Digest (Applied Physics Laboratory), vol. 25, no. 4, pp. 348-355, 2004.

[2] C. Situ, M. H. Mooney, C. T. Elliott, and J. Buijs, "Advances in surface plasmon resonance biosensor technology towards high-throughput, food-safety analysis," TrAC Trends in Analytical Chemistry, vol. 29, no. 11, pp. 1305-1315, 2010.

[3] J. Zhou, Q. Qi, C. Wang et al., "Surface plasmon resonance (SPR) biosensors for food allergen detection in food matrices," Biosensors and Bioelectronics, vol. 142, Article ID 111449 , 2019.

[4] A. Dhawan, M. Canva, and T. Vo-Dinh, "Narrow groove plasmonic nano-gratings for surface plasmon resonance sensing," Optics Express, vol. 19, no. 2, pp. 787-813, 2011.

[5] B. Ng, S. M. Hanham, J. Wu et al., "Broadband terahertz sensing on spoof plasmon surfaces," ACS Photonics, vol. 1, no. 10, pp. 1059-1067, 2014.

[6] A. Alipour, A. Farmani, and A. Mir, "High sensitivity and tunable nanoscale sensor based on plasmon-induced transparency in plasmonic metasurface," IEEE Sensors Journal, vol. 18, no. 17, pp. 7047-7054, 2018. 
[7] V. V. Bulgakova, V. V. Gerasimov, B. G. Goldenberg, A. G. Lemzyakov, and A. M. Malkin, "Study of terahertz spoof surface plasmons on subwavelength gratings with dielectric substance in grooves," Procedia Engineering, vol. 201, pp. 14-23, 2017.

[8] J. F. Federici, B. Schulkin, F. Huang et al., "THz imaging and sensing for security applications-explosives, weapons and drugs," Semiconductor Science and Technology, vol. 20, no. 7, pp. S266-S280, 2005.

[9] A. G. Davies, A. D. Burnett, W. Fan, E. H. Linfield, and J. E. Cunningham, "Terahertz spectroscopy of explosives and drugs," Materials Today, vol. 11, no. 3, pp. 18-26, 2008.

[10] J. F. O'Hara, W. Withayachumnankul, and I. Al-Naib, "A review on thin-film sensing with terahertz waves," Journal of Infrared, Millimeter, and Terahertz Waves, vol. 33, no. 3, pp. 245-291, 2012.

[11] T. Akalin, A. Treizebre, and B. Bocquet, "Single-wire transmission lines at terahertz frequencies," IEEE Transactions on Microwave Theory and Techniques, vol. 54, no. 6, pp. 27622767, 2006.

[12] M. Islam, S. J. M. Rao, G. Kumar, B. P. Pal, and D. Roy Chowdhury, "Role of resonance modes on terahertz metamaterials based thin film sensors," Scientific Reports, vol. 7, no. 1, p. $7355,2017$.

[13] K. A. Willets and R. P. Van Duyne, "Localized surface plasmon resonance spectroscopy and sensing," Annual Review of Physical Chemistry, vol. 58, no. 1, pp. 267-297, 2007.

[14] J. Homola, S. S. Yee, and G. Gauglitz, "Surface plasmon resonance sensors: review," Sensors and Actuators B: Chemical, vol. 54, no. 1-2, pp. 3-15, 1999.

[15] D. Shankaran, K. Gobi, and N. Miura, "Recent advancements in surface plasmon resonance immunosensors for detection of small molecules of biomedical, food and environmental interest," Sensors and Actuators B: Chemical, vol. 121, no. 1, pp. 158-177, 2007.

[16] T. W. Ebbesen, H. J. Lezec, H. F. Ghaemi, T. Thio, and P. A. Wolff, "Extraordinary optical transmission through subwavelength hole arrays," Nature, vol. 391, no. 6668, pp. 667-669, 1998.

[17] W.-H. Yeh, J. Kleingartner, and A. C. Hillier, "Wavelength tunable surface plasmon resonance-enhanced optical transmission through a chirped diffraction grating," Analytical Chemistry, vol. 82, no. 12, pp. 4988-4993, 2010.

[18] A. Ryabchun, M. Wegener, Y. Gritsai, and O. Sakhno, "Novel effective approach for the fabrication of PDMS-based elastic volume gratings," Advanced Optical Materials, vol. 4, no. 1, pp. 169-176, 2016.

[19] P. Gutruf, E. Zeller, S. Walia, S. Sriram, and M. Bhaskaran, "Mechanically tunable high refractive-index contrast TiO2PDMS gratings," Advanced Optical Materials, vol. 3, no. 11, pp. 1565-1569, 2015.

[20] B. Sheng, L. Luo, Y. Huang, G. Chen, H. Zhang, and S. Zhuang, "Tailorable elastomeric grating with tunable groove density gradient," IEEE Photonics Journal, vol. 9, no. 5, pp. 1-6, 2017.

[21] W. Liu, Y. Shen, G. Xiao, X. She, J. Wang, and C. Jin, "Mechanically tunable sub-10 nm metal gap by stretching PDMS substrate," Nanotechnology, vol. 28, no. 7, Article ID 075301, 2017.

[22] M. Nazarov, F. Garet, D. Armand, A. Shkurinov, and J.-L. Coutaz, "Surface plasmon $\mathrm{THz}$ waves on gratings," Comptes Rendus Physique, vol. 9, no. 2, pp. 232-247, 2008.

[23] I. S. Spevak, A. A. Kuzmenko, M. Tymchenko et al., "Surface plasmon-polariton resonance at diffraction of $\mathrm{THz}$ radiation on semiconductor gratings," Low Temperature Physics, vol. 42, no. 8, pp. 698-702, 2016.
[24] S. Lin, K. Bhattarai, J. Zhou, and D. Talbayev, "Giant THz surface plasmon polariton induced by high-index dielectric metasurface," Scientific Reports, vol. 7, no. 1, p. 9876, 2017.

[25] S. Alfihed, M. H. Bergen, J. F. Holzman, and I. G. Foulds, “A detailed investigation on the terahertz absorption characteristics of polydimethylsiloxane (PDMS)," Polymer, vol. 153, pp. 325-330, 2018.

[26] W. L. Barnes, A. Dereux, and T. W. Ebbesen, "Surface plasmon subwavelength optics," Nature, vol. 424, no. 6950, pp. 824-830, 2003.

[27] S. Nair, C. Escobedo, and R. G. Sabat, "Crossed surface relief gratings as nanoplasmonic biosensors," ACS Sensors, vol. 2, no. 3, pp. 379-385, 2017.

[28] J.-Y. Jing, Q. Wang, W.-M. Zhao, and B.-T. Wang, "Longrange surface plasmon resonance and its sensing applications: a review," Optics and Lasers in Engineering, vol. 112, pp. 103-118, 2019.

[29] Y. Si, J. Lao, X. Zhang et al., "Electrochemical plasmonic fiberoptic sensors for ultra-sensitive heavy metal detection," Journal of Lightwave Technology, vol. 37, no. 14, pp. 34953502, 2019.

[30] S. Mariani and M. Minunni, "Surface plasmon resonance applications in clinical analysis," Analytical and Bioanalytical Chemistry, vol. 406, no. 9-10, pp. 2303-2323, 2014.

[31] K. Lin, Y. Lu, J. Chen, R. Zheng, P. Wang, and H. Ming, "Surface plasmon resonance hydrogen sensor based on metallic grating with high sensitivity," Optics Express, vol. 16, no. 23, pp. 18599-18604, 2008.

[32] C. Hu and D. Liu, "High-performance grating coupled surface plasmon resonance sensor based on Al-Au Bimetallic layer," Modern Applied Science, vol. 4, no. 6, p. 8, 2010.

[33] M. G. Moharam, T. K. Gaylord, D. A. Pommet, and E. B. Grann, "Stable implementation of the rigorous coupledwave analysis for surface-relief gratings: enhanced transmittance matrix approach," Journal of the Optical Society of America A, vol. 12, no. 5, pp. 1077-1086, 1995.

[34] M. Yin, S. Tang, and M. Tong, "The application of terahertz spectroscopy to liquid petrochemicals detection: a review," Applied Spectroscopy Reviews, vol. 51, no. 5, pp. 379-396, 2016.

[35] Y. S. Jin, G. J. Kim, C.-H. Shon, S. G. Jeon, and J. I. Kim, "Analysis of petroleum products and their mixtures by using terahertz time domain spectroscopy," Journal of the Korean Physical Society, vol. 53, no. 4, pp. 879-1885, 2008.

[36] G.-J. Kim, S.-G. Jeon, J.-I. Kim, and Y.-S. Jin, “Terahertz time domain spectroscopy of petroleum products and organic solvents," in Proceedings of the 2008 33rd International Conference on Infrared, Millimeter and Terahertz Waves, Pasadena, CA, USA, September 2008.

[37] Y. Zhang, X. Zhang, S. Li et al., "A broadband THz-TDS system based on DSTMS emitter and LTG InGaAs/InAlAs photoconductive antenna detector," Scientific Reports, vol. 6, no. 1, p. 26949, 2016.

[38] M. Querry, Optical Constants of Minerals and Other Materials from the Millimeter to the Ultraviolet, Chemical Research Development and Engineering Center Aberdeen Proving Groundmd, USA, 1987.

[39] M. A. Ordal, R. J. Bell, R. W. Alexander, L. L. Long, and M. R. Querry, "Optical properties of $\mathrm{Au}, \mathrm{Ni}$, and $\mathrm{Pb}$ at submillimeter wavelengths," Applied Optics, vol. 26, no. 4, pp. 744-752, 1987.

[40] C. Caucheteur, M. Loyez, Á. González-Vila, and R. Wattiez, "Evaluation of gold layer configuration for plasmonic fiber grating biosensors," Optics Express, vol. 26, no. 18, pp. 24154-24163, 2018. 
[41] S. Nagai, T. Hayashi, and A. Sanada, "Measurements of anomalous skin effect in $1 \mathrm{THz}$ band," in Procedings of the 2013 IEEE MTT-S International Microwave Symposium Digest (MTT), pp. 1-3, Seattle, WA, USA, June 2013.

[42] X. Qiu, X. Chen, F. Liu, B.-O. Guan, and T. Guo, "Plasmonic fiber-optic refractometers based on a high Q-factor Amplitude interrogation," IEEE Sensors Journal, vol. 16, no. 15, pp. 5974-5978, 2016.

[43] H. Hori, K. Tawa, K. Kintaka, J. Nishii, and Y. Tatsu, "Influence of groove depth and surface profile on fluorescence enhancement by grating-coupled surface plasmon resonance," Optical Review, vol. 16, no. 2, pp. 216-221, 2009.

[44] S. T. Koev, A. Agrawal, H. J. Lezec, and V. A. Aksyuk, "An efficient large-area grating coupler for surface plasmon polaritons," Plasmonics, vol. 7, no. 2, pp. 269-277, 2012. 

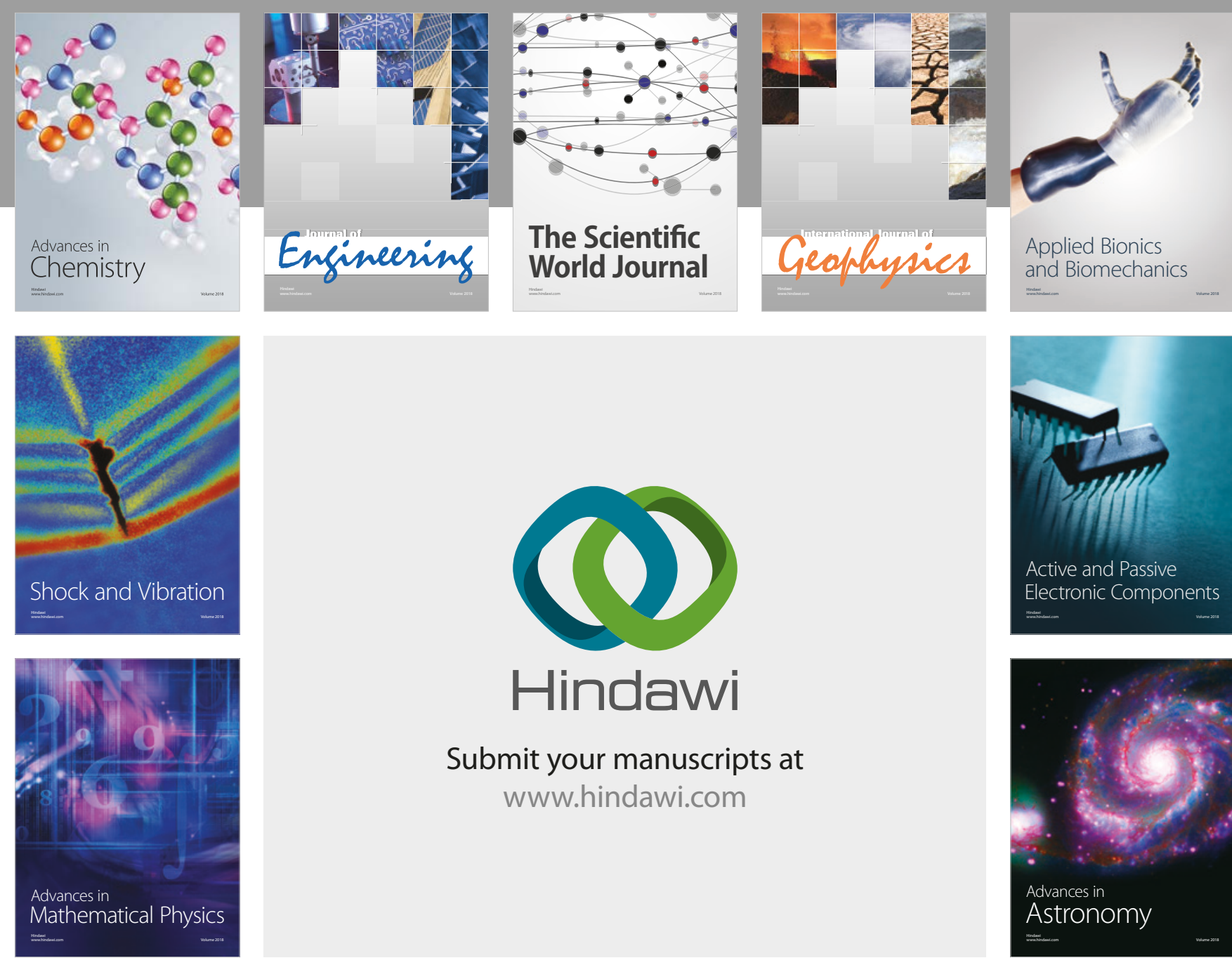

Submit your manuscripts at

www.hindawi.com

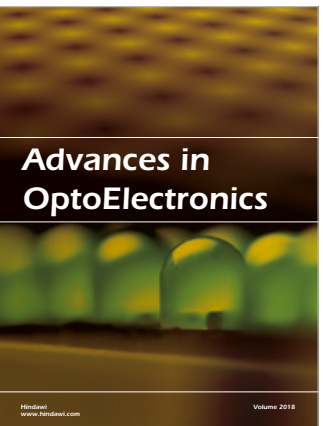

\section{Rotcting Machinery}
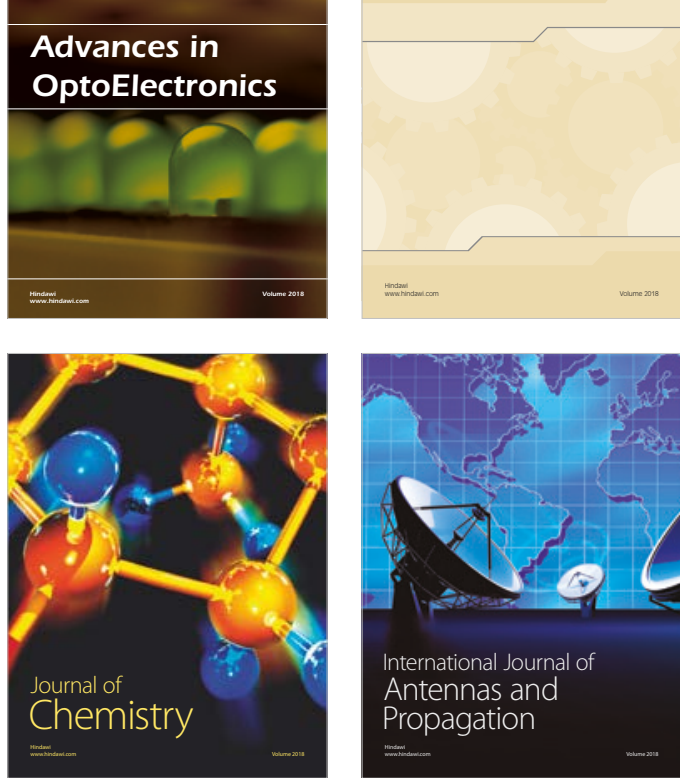

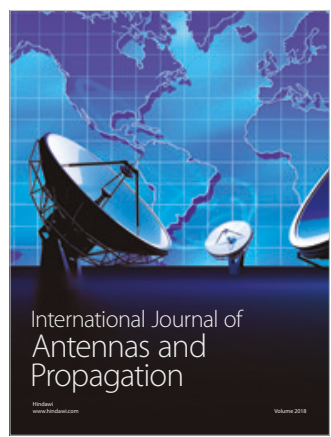

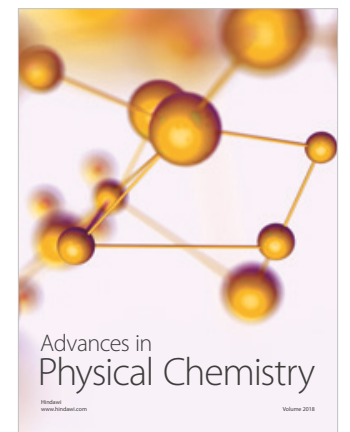

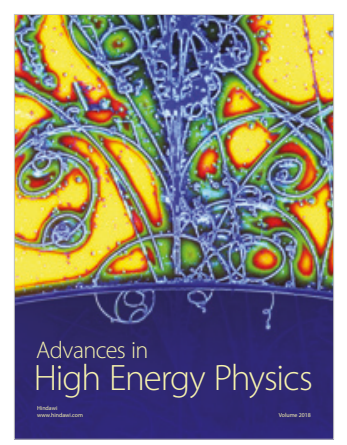

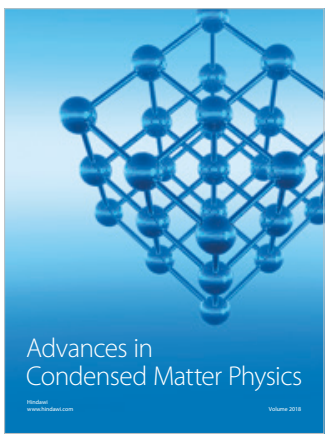

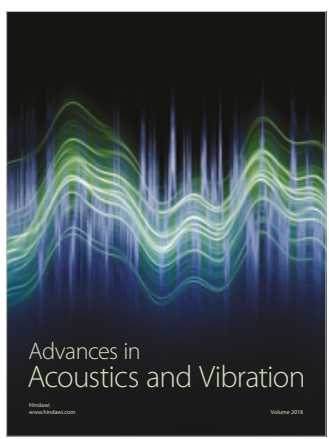

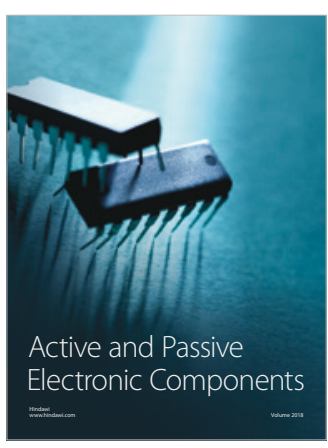
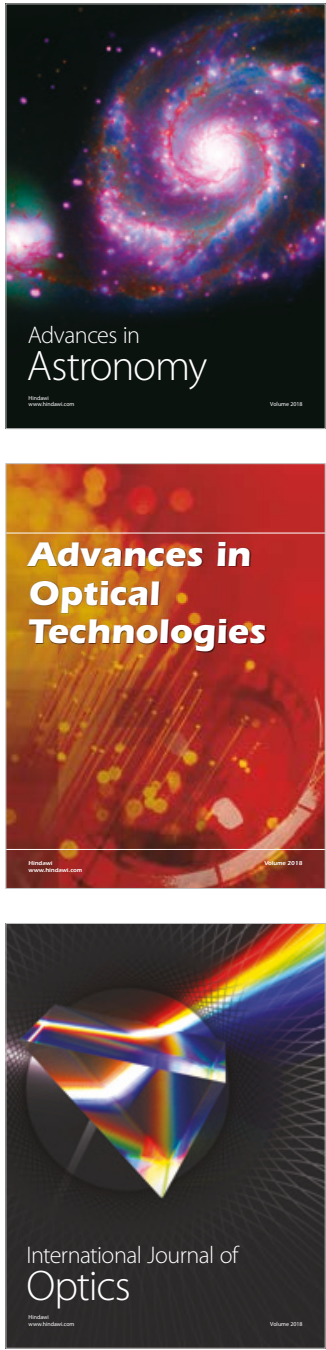